\title{
EXPLORATION DES VOIES SEMINALES PAR L'ETUDE DU PLASMA SEMINAL
}

\author{
A. Clavert \\ Groupe de recherche en Andrologie, Faculté de Médecine de Strasbourg, 67000, Strasbourg
}

\section{INVESTIGATION OF THE MALE ACCESSORY GLANDS BY BIOCHE-} MICAL ANALYSIS OF SEMINAL PLASMA. The biochemical analysis of seminal plasma provides information on the functional status of the male accessory glands. A semen analysis evaluates the relative contribution of these various secretions as well as the presence of leukocytes. Measurements of specific markers for prostatic, vesicular and epididymal secretions are indicative of glandular function, and similarly markers of inflammatory response(s) can presage infections. Furthermore, such assays may be diagnostic of the level of an obstruction or the severity of glandular dysfunction. Keys words : Seminal plasma, prostate, seminal vesicle, epididymis, inflammation. Andrologie, 1992, 2 : 68-69.

Le plasma séminal se constitue après l'éjaculation par le mélange des sécrétions des différentes glandes séminales. Il est de ce fait possible, grâce à son analyse, d'avoir des renseignements sur l'activité de ces glandes. Il est également possible de recueillir des indices pouvant être utilisés pour confirmer des diagnostics ou diagnostiquer des processus pathologiques cliniquement muets.

Cette exploration prend une importance d'autant plus grande que les fonctions du plasma séminal(PS) résultent de l'interaction des sécrétions entre elles ; ainsi la réduction de la sécrétion de l'une d'entre elles aura des conséquences sur la fertilité.

\section{REMARQUES PRELIMINAIRES.}

La composition du PS dépend en partie du déroulement de l'éjaculation au moment du prélèvement; il est de ce fait important de faire un interrogatoire pour éliminer une éjaculation rétrograde partielle.

Les sécrétions recueillies résultent de processus d'excrétion et de sécrétion qui se déroulent avant l'expulsion. Les vésicules séminales stockent les sécrétions alors que les glandes prostatiques excrètent pendant la phase d'excitation sexuelle peu de temps avant l'éjaculation. La fréquence des éjacu!ations joue un rôle important sur le volume éjaculé.

Après l'éjaculation, le sperme est coagulé, puis il se liquéfie. Ce phénomène résulte de l'action des enzymes, le plus souvent prostatiques, sur le coagulum, essentiellement vésiculaire. Pendant cette évolution la composition en sucres, lipides et protéines se modifie (4), ainsi les résultats varient suivant le moment où les dosages sont réalisés.

En pratique, cela implique de prendre des précautions:

- interroger le patient pour connaitre la fréquence des rapports et la date du dernier rapport,

- demander s'il n'y a pas eu de pertes au moment du prélèvement,

-faire préciser par le laboratoire combien de temps après l'éjaculation le sperme a été centrifugé puis congelé (la congélation est indispensable pour arrêter les réactions enzymatiques)

-se souvenir au moment de l'interprétation que le plasma séminal n'est qu'un instantané et un reflet quelquefois lointain de ce qui se passe dans le tractus génital.

En général, une demi-heure après l'éjaculation, après avoir noté le volume, le $\mathrm{pH}$, la couleur et la viscosité, le sperme est centrifugé, puis immédiatement congelé.

\section{L'ANALYSE DU PLASMA SEMINAL.}

\section{Le spermogramme.}

Cet examen permet de fournir des renseignements précieux. En effet on sait que :

- les sécrétions prostatiques sont très acides $(\mathrm{pH}$ 6,4), liquides, blanchâtres,

- les sécrétions vésiculaires par contre sont basiques ( $\mathrm{pH} 8,4$ environ), visqueuses et jaunâtres .On sait également que la viscosité dépend en grande partie de la sécrétion vésiculaire et que la vitesse de liquéfaction résulte de la concentration d'enzymes prostatiques.

Le volume total, le pH, la couleur, la viscosité initiale et la vitesse de liquéfaction donnent une bonne idée de l'importance relative des sécrétions prostatiques et vésiculaires.
L'étude cytologique des cellules rondes.

L'étude des cellules rondes est une étape capitale pour comprendre certains processus pathologiques ; dans certains cas il est possible dès le spermocytogramme de faire le diagnostic de polynucléaires, mais le plus souvent des techniques adaptées doivent être utilisées.

Cette recherche se fait par la réaction histoenzymatique de la peroxydase.La recherche des macrophages est également possible par histoenzymologie, par la mise en évidence des phospholipases non spécifiques (5).

\section{L'éjaculation fractionnée.}

Cette technique permet de savoir si les polynucléaires proviennent de la prostate, de la vésicule séminale, ou des épididymes.

\section{Dosage des marqueurs glandulaires.}

Le marqueur est une substance qui est spécifique d'une sécrétion, son dosage permet suivant sa physiologie de savoir si la sécrétion est présente dans l'éjaculat et dans certains cas de déterminer le volume relatif de la sécrétion.

- Les marqueurs des glandes urétrales:

Il n'existe pas à l'heure actuelle de marqueur utilisable en routine ; l'étude de certaines mucines est prometteuse.

\section{- Les marqueurs de la prostate:}

Les marqueurs le plus souvent utilisés sont le zinc, l'acide citrique, et l'activité de la phosphatase acide prostatique. Pour évaluer la sécrétion globale l'un de ces dosages est suffisant. Mais la prostate est un organe unique constitué par plusieurs glandes ayant des sécrétions différentes. Dans certains cas, il est possible de montrer qu'il existe des dissociations entre la concentration en zinc et l'activité phosphatasique (3). Ces observations sont rares et n'apportent pas de renseignements décisifs pour le diagnostic.

\section{- Les marqueurs vésiculaires :}

Le marqueur le plus fidèle et le plus discriminant est et reste le fructose. La quantité de fructose d'un éjaculat est proportionnelle à la surface d'épithélium fonctionnel. Les protéines proviennent pour l'essentiel des vésicules, le dosage des protéines totales peut confirmer celui du fructose; dans certains cas rares, il existe des dissociations. L'électrophorèse permet de 
metre en évidence des bandes correspondant aux protéines spécifiques de la glande (1).

- Les marqueurs épididvmaires:

La carnitine, la glycérophophorylcholine, et l'activité de l'alpha-glycosidase sont des marqueurs de la présence de la sécrétion épididymaire ; ils sont excrétés au niveau de la partie supérieure de la glande, puis réabsorbés ou tranformés lors de leur passage dans la partie inférieure du tractus. En outre, des concentrations faibles de carnitine proviennent de la vésicule séminale. Ainsi il n'est pas possible de trouver une relation entre la concentration en carnitine et l'activité de l'organe, par contre leur dosage est interéssant afin de distinguer les azoospermies excrétoires des azoospermies sécrétoires (6).

\section{- Les marqueurs de l'inflammation:}

Au spermogramme, il est possible de mettre en évidence la présence de polynucléaires et, ou de macrophages; ces cellules sont des indices d'un processus inflammatoire. Il est possible de confirmer en recherchant des marqueurs biochimiques de l'inflammation comme les Ig A épithéliaux qui apparaissent tôt dans le processus inflammatoire (3).

L'électrophorèse des protéines peut mettre en évidence l'augmentation des protéines sériques et ainsi visualiser l'existence d'un transudat. Dans certains cas, il n'existe qu'une augmentation de l'albumine, qui prend alors une signification inflammatoire plus probante.

\section{LES DIAGNOSTICS}

La modification d'un seul paramètre du plasma séminal ne peut être prise en considération pour réaliser un diagnostic. Par contre il constitue un signe d'appel qui doit être confirmé.
Diagnostic du niveau d'obstruction dans les azoospermies excrétoires.

- Obstruction des canaux excrétoires: Volume inférieur à $1 \mathrm{ml}, \mathrm{pH}$ acide < à 7 , plasma blanchâtre, liquide, fructose nul, carnitine abaissée, Zn et phosphatase acide élevés.

- Obstruction bilatérale des déférents/des épididymes: Volume, $\mathrm{pH}$, couleur, viscosité, $\mathrm{Zn}$, Phosphatases acides et fructose normaux, Carnitine, GPC, alpha glucosidase effondrées

\section{Localisation d'une atteinte glandulaire.}

La modification d'au moins deux paramètres spécifiques d'une glande permet de suspecter un processus au niveau de celle-ci.

- Prostate : Viscosité augmentée, avec liquéfaction retardée, voire absente, $\mathrm{pH}$ alcalin, $\mathrm{Zn}$, phos phatase acide diminués.

- Vésicule séminale : Viscosité diminuée, $\mathrm{pH}$ acide, Fructose diminué, protéines totales dimi nuées.

\section{Diagnostic différentiel entre séquelles d'infec- tion et infection probable en cours}

Lorsque la sécrétion d'une glande est réduite et qu'en outre il a été retrouvé des polynucléaires, des IgA et/ou des protéines sériques augmentées, il faut suspecter l'existence d'un processus évolutif.

\section{CONCLUSIONS}

La procréation médicalement assistée a besoin de spermatozoïdes ayant une maturation complète, et d'un plasma séminal non infecté, même par des germes saprophytes. Ainsi il est indispensable de préparer les hommes avant de se lancer dans ces techniques, et de traiter toutes lésions du tractus génital même si elles sont infra cliniques, d'où le besoin d'affiner le diagnostic. L'explora- tion des voies séminales par l'étude du plasma séminal prend ainsi toute sa valeur.

\section{REFERENCES}

1 - Clavert A., Cranz C., Bollack C. : Fonctions of the seminal vesicle. Andrologia 1990,22 : 185-192.

2 - Clavert A., Montagnon D., Brun B. : Pathological seminal plasma proteins analysed by acetate electrophoresis. Arch. of Andrology., 1981, 6: 53-60.

3- Cranz C., Montagnon D., Becker N., Clavert A. : Pathological seminal fuid and IgA. Infertility., $1987,10: 173-183$.

4 - Montagnon D., Brun B., Clavert A. : Vesicular proteins and their relationships with reducing sugars. Prog.Reprod. Biol. Med, 1985, 12 : 64-79.

5 - Nishima T., Teroshida Y., Hattort T., Satohi Yoshida M., Akimoto M. : Study of macrophages in prostatic fluid from non bacterial prostatitis patients. Urol. Intern, 1991, $32: 15-17$.

6 - Soufir J.C., Jeulin C. : Origin of L. Carnitin of human seminal plasma. Prog. Reprod. Biol. Med. $1985,12: 4-7$.

RESUME : L'analyse du plasma séminal donne des informations sur l'état fonctionnel des glandes du tractus seminal. Le spermogramme évalue l'importance des sécrétions, et la présence éventuelle de polynucléaires ; le dosage des marqueurs permet d'avoir une idée sur le niveau d'activité de chaque glande, et les marqueurs de l'inflammation attirent l'attention sur une infection éventuelle, ainsi le niveau d'une obstruction peut être diagnostiqué et l'étendue des lésions glandulaires peut être évaluée. Mots clés : Plasma séminal - prostate - vésicule séminale - epididyme - inflammation. Andrologie, 1992, 2 : 67-69. 